\title{
The response to high magnetic fields of the Vacuum Phototriodes for the Compact Muon Solenoid endcap electromagnetic calorimeter
}

\author{
K W Bell ${ }^{1}, \mathrm{R}$ M Brown ${ }^{1}$, D J A Cockerill ${ }^{1}$, P S Flower ${ }^{1}$, P R Hobson ${ }^{2}$, D C Imrie ${ }^{2}$, B W Kennedy ${ }^{1}$, \\ A L Lintern ${ }^{1}$, O Sharif ${ }^{2}$, M Sproston $^{1}$, J H Williams ${ }^{1}$ \\ ${ }^{1}$ CLRC - Rutherford Appleton Laboratory, Chilton, Didcot, OX11 0QX UK \\ ${ }^{2}$ Brunel University, Department of Electronic and Computer Engineering, Uxbridge, UB8 3PH, UK
}

\begin{abstract}
The endcap electromagnetic calorimeter of the Compact Muon Solenoid (CMS) detects particles with the dense fast scintillator lead tungstate $\left(\mathrm{PbWO}_{4}\right)$. Due to the low light yield of this scintillator photodetectors with internal gain are required. Silicon avalanche photodiodes cannot be used in the endcap region due to the intense neutron flux. Following an extensive R\&D programme $26 \mathrm{~mm}$ diameter single-stage photomultipliers (vacuum phototriodes) have been chosen as the photodetector in the endcap region. The first 1400 production devices are currently being evaluated following recent tests of a pre-production batch of 500 tubes. Tubes passing our acceptance tests have responses, averaged over the angular acceptance of the endcap calorimeter, corresponding to the range 20 to 55 electrons per $\mathrm{MeV}$ deposited in $\mathrm{PbWO}_{4}$. These phototriodes operate, with a typical gain of 10 , in magnetic fields up to $4 \mathrm{~T}$.
\end{abstract}

\section{Introduction}

The Compact Muon Solenoid (CMS) detector which will operate at the Large Hadron Collider is a large general purpose particle detector [1] In order to provide excellent energy resolution the barrel and endcap electromagnetic calorimeters use the dense fast scintillator lead tungstate $\left(\mathrm{PbWO}_{4}\right)$. Due to the low light yield of this scintillator photodetectors with internal gain are required. In the barrel region silicon avalanche photodiodes will be used, but these cannot be used in the endcap region due to the intense neutron flux. Following an extensive R\&D programme $26 \mathrm{~mm}$ diameter single-stage photomultipliers (vacuum phototriodes) have been chosen as the photodetector in the endcap region. The first 1400 production devices are currently being evaluated following recent successful tests of a pre-production batch of 500 tubes.

\section{Operating principles}

A vacuum phototriode (VPT) is a single stage photomultiplier tube with a fine mesh transmissive anode and a solid high-gain dynode. The gain is dependent upon the transparency of the mesh and the secondary electron gain coefficient of the dynode. For the tubes described here, a transparency of around 50\% and a typical secondary emission gain of 20 result in a nominal gain at $0 \mathrm{~T}$ of about 9 . For a constant operating potential the gain of the tube is a function of field strength and angle to the field [2] [3]. At a constant angle to the field tubes show a rapid fall in gain with increasing field, reaching a plateau of $80 \%$ of the zero field gain around 1T. In CMS the devices must operate at the full field of the experiment, 4T.

\section{CMS VPT characteristics}

Five hundred tubes were delivered by RIE [4] during 2001 and were evaluated at Brunel University and CLRC Rutherford Appleton Laboratory. In particular their characteristics in magnetic fields were studied in detail. At RAL the gain of the tubes is measured as a function of field strength up to a maximum axial field of $1.8 \mathrm{~T}$, and the variation of gain with angle within a constant field of $1.8 \mathrm{~T}$ is also determined. Tubes show a complicated oscillatory response at a fixed field as they are tilted away from axial alignment (figure 1) It has been shown that this is a fundamental feature, which depends only on the construction details in particular the mesh transparency and mesh thickness, and is highly reproducible from tube to tube [5].

\footnotetext{
* Corresponding author: EMAIL Peter.Hobson@brunel.ac.uk, FAX +44(0)1895 272391, TEL +44(0)1895 203203
} 

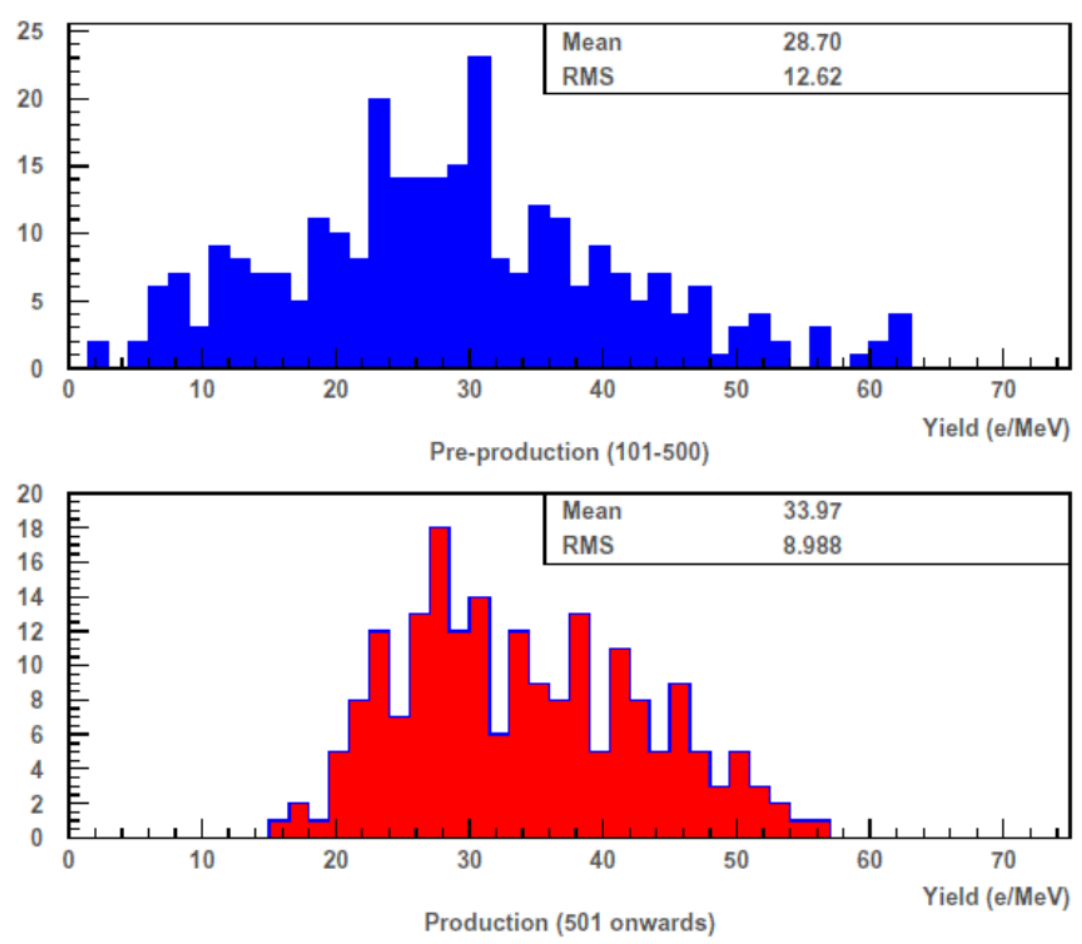

Figure 3 Measured response of the RIE tubes averaged over the angular range $8^{\circ}$ to $25^{\circ}$ in a $1.8 \mathrm{~T}$ field. The measured anode response has been converted to give the electron yield per $\mathrm{MeV}$ deposited in an endcap $\mathrm{PbWO}_{4}$ crystal.

\section{Summary}

Following successful evaluation of 500 pre-production tubes from RIE we are now taking delivery of the first batches of the 15000 tubes destined for the CMS endcap electromagnetic calorimeters. We have demonstrated that vacuum phototriodes, with a gain of about 10 at $15^{\circ}$ to a magnetic field of $4 \mathrm{~T}$ can be commercially produced in quantity. These new devices are superior in many respects to those previously obtainable, notably in having high gain at high magnetic field strength, radiation tolerance, and stability at large photocurrents. Other applications that need these characteristics could benefit from these new photodetectors.

\section{Acknowledgement}

This research is supported by the Particle Physics and Astronomy Research Council, UK and in part by the European Commission (INTAS-CERN scheme 99-424).

\section{References}

1 The Compact Muon Solenoid Technical Proposal, CERN/LHCC 94-38, 1994

2 K.W. Bell et al, Nucl.Instr. and Meth A469 (2001) 29

3 M Apollonio et al, Nucl.Instr. and Meth A484 (2002) 287

4 National Research Institute Electron, Moisa Toreza Ave., 68, 194223 St Petersburg, Russia

$5 \mathrm{~J}$ E Bateman, "The operation of vacuum phototriodes in a non-axial magnetic field - a Monte Carlo study" CMS NOTE 1999/032 